\title{
Development and Evaluation of the MAINTAIN Instrument, Selecting Patients Suitable for Secondary or Tertiary Preventive Manual Care - The Nordic Maintenance Care Program
}

\section{Andreas Eklund ( $\nabla$ andreas.eklund@ki.se )}

Institute of Environmental Medicine (IMM), Karolinska Institutet https://orcid.org/0000-0002-21627537

\section{Per Palmgren}

Management and Ethics (LIME), Karolinska Institutet

\section{Ulf Jakobsson}

Department of Clinical Sciences Malmö, Lund University Iben Axén

The Institute of Environmental Medicine (IMM), Karolinska Institutet

\section{Research Article}

Keywords: MAINTAIN, clinical instrument, low back pain, stratified care, chiropractic, prevention, maintenance care, manual treatment, effect, secondary prevention, tertiary prevention

Posted Date: September 7th, 2021

DOI: https://doi.org/10.21203/rs.3.rs-860986/v1

License: (c) (i) This work is licensed under a Creative Commons Attribution 4.0 International License. Read Full License

Version of Record: A version of this preprint was published at Chiropractic \&amp; Manual Therapies on March 17th, 2022. See the published version at https://doi.org/10.1186/s12998-022-00424-6. 


\section{Abstract}

\section{Background}

Chiropractic Maintenance Care (MC) has been found to be effective for patients classified as dysfunctional (high pain severity, marked interference with everyday life due to pain, high affective distress, low perception of life control, and low activity levels) by the Swedish equivalent of the West Haven-Yale Multidimensional Pain Inventory (MPI-S). Although displaying good psychometric properties such as validity and reliability, the instrument was not designed to be used in clinical practice to screen patients for stratified care pathways. To effectively be able to screen for individuals suitable for $M C$, the aim was to develop a clinical instrument with the intent of identifying dysfunctional patients with acceptable sensitivity, specificity, and discriminant ability.

\section{Methods}

Data from 249 patients with a complete MPI dataset from an RCT that investigated the effect and costeffectiveness of MC with a 12-month follow-up was used in this cross-sectional analysis. The MPI's data was used to develop a short screening instrument to identify dysfunctional patients, with a summary measure, based on the original instrument. Different cutoffs were considered with regards to sensitivity, specificity, and discriminant ability and compared to the original instrument's classification of dysfunctional patients. The instrument was then tested in 3 other existing datasets to assess validity across populations.

\section{Results}

Using an explorative approach, the MAINTAIN instrument with 10 questions (0-6 Likert responses) with 5 dimensions (pain severity, interference, life control, affective distress, and support) was developed, generating an algorithm-based score ranging from -12 to 48. Reporting a MAINTAIN score of 18 or higher, 146 out of the 249 patients were classified as dysfunctional with $95.8 \%$ sensitivity and $64.3 \%$ specificity. At a score of 22 or higher, 109/249 were classified as dysfunctional with $81.1 \%$ sensitivity and $79.2 \%$ specificity. Discriminant ability (area under the curve (AUC)) was estimated to 0.87 (95\% Cl: 0.83, 0.92; $p$ $<0.001)$ and Youden's index was highest $(0.70)$ at a score of 20 . The discriminant ability is similar and acceptable across populations with minor differences in optimal thresholds for identifying dysfunctional individuals.

\section{Conclusion}

The MAINTAIN instrument had an acceptable performance with regards to identifying dysfunctional patients and may be used as a decision aid in clinical practice. By using 2 thresholds, patients can be categorized into "low probability (-12 to 17)", "moderate probability (18 to 21$)$ ", and "high probability (22 to 48)" of having a good outcome from maintenance care for Low Back Pain (LBP).

\section{Trial registration}


Clinical trials.gov; NCT01539863; registered February 28, 2012;

https://clinicaltrials.gov/ct2/show/NCT01539863

\section{Background}

Non-specific Low Back Pain (LBP) is a highly prevalent condition affecting a large part of the adult population with major consequences worldwide. It is considered one of the biggest economic burdens in western societies (1). The lifetime prevalence worldwide has been estimated to be $80-85 \%$ and the condition is often characterized by recurrent episodes (2). According to the Global Burden of Disease Study, LBP results in more years lived with disability than any other disease in the world (2).

For a highly disabling recurrent and costly condition like LBP, it seems logical to invest in preventive strategies to mitigate and minimize the consequences. However, the evidence for effective interventions aimed at preventing LBP is scarce and until now, only exercise, and exercise in combination with education, have been shown empirically to be effective (3).

Chiropractic MC is described as a long-term management strategy for musculoskeletal disorders, introduced when optimum treatment benefit has been reached after an initial care plan. The aim is to prevent future episodes and deterioration by treating the patient at regular intervals, regardless of symptoms (4-18). In an ambitious effort, researchers across the Scandinavian countries have systematically explored and investigated indications, content, and frequency of $\mathrm{MC}$ in a series of research projects (8-22). Commonly, MC patients are selected based on their previous history of pain and the effectiveness of the initial care plan. Selected MC patients are commonly scheduled with 1-3 months intervals and are treated with manual therapy, along with individual exercises and lifestyle advice (18).

Based on this knowledge, a pragmatic randomized clinical trial was designed to investigate the effectiveness of MC in patients with recurrent and persistent LBP (19). The trial found that the MC-group had 12.8 (95\% Cl: 10.1, 15.5; $\mathrm{p}:<0.001$ ) fewer days with bothersome LBP over a year compared to the control group (20). Although more effective, the number of visits was higher in the MC-group with 1.7 (95\% Cl: $1.8,2.1 ; \mathrm{p}:<0.001)$ more treatments during the 52-week study period (20). There was a large variability in the data, suggesting that there may be subgroups of patients who experienced fewer days of pain and fewer visits than others.

Psychological $(23,24)$, behavioural $(25)$ and social characteristics (26) of LBP patients are important in the transition from acute to recurrent and persistent pain states (27-33). In line with the bio-psychosocial model, the leading theoretical framework underpinning the management of LBP $(26,34-36)$ clinicians would be expected to consider cognitive processes, psychological and behavioural dimensions of the pain experienced when managing patients with pain. Based on the cognitive-behavioural conceptualization of pain, The West Haven-Yale Multidimensional Pain Inventory (MPI) was developed to capture the perceptions and consequences of living with chronic pain (37). 
The original instrument has been used to identify three clusters/subgroups of patients (38) and has been shown to be reliable, valid, and useful in outcome-based research $(39,40)$. The three different subgroups are defined as Adaptive Copers (AC), Interpersonally Distressed (ID), and Dysfunctional (DYS). Individuals in the AC group are characterized by low pain severity, low interference with everyday life due to pain, low life distress, a high activity level, and a high perception of life control and have the best prognosis with the lowest risk for long-term sick leave (41). Individuals in the ID group are characterized by dysfunctional behaviours such as low levels of social support, low levels of solicitous and distracting responses from significant others, and high scores on punishing responses compared to the DYS and AC patients (41). Individuals in the DYS subgroup are characterized by having high pain severity, marked interference with everyday life due to pain, high affective distress, low perception of life control, and low activity levels and have the worst prognosis along with the highest risk of long-term sickness absence (41). The identification of these subgroups has been used in clinical settings to investigate a diversity of conditions such as neck pain and LBP (41-43), temporomandibular disorders (44), headaches (45), fibromyalgia (46), and cancer pain (47) and are associated with different clinical outcomes.

To suggest that the outcome of MC may be affected by psychological characteristics such as those identified with the MPI instrument is an appealing idea and could lead to a tailored approach and better outcomes in the prevention of LBP. In a secondary analysis of the data from the RCT $(21,22)$, it was found that patients with a less favourable psychological profile (DYS subgroup) reported better outcomes from the MC approach (30.0 days less with pain, 95\% Cl: -36.6, -23.4). Surprisingly, the effect of MC within the DYS subgroup was achieved at an equal number of visits compared to the control group. On the other hand, patients within the AC group who received $M C$ reported more days with pain (10.7 more days, $95 \% \mathrm{Cl}: 4.0,17.5)$ while also receiving a higher number of visits compared to the control group (3.5 more visits, $95 \% \mathrm{Cl}: 1.8,5.3)$.

These results may change the way $\mathrm{MC}$ is delivered in clinical practice as we can now identify subgroups of patients where $\mathrm{MC}$ is most effective. At the same time, it becomes clear that for a specific group of patients, the AC group, MC should not be recommended. In the AC group, the effect of $M C$ is, at best, equally effective or in a worst-case scenario, results in more days with LBP while at the same time it results in more visits to the chiropractor compared to the control group.

Although valid and reliable, the Swedish version of the MPI instrument (MPI-S) was not designed to be used in daily clinical practice but rather as a comprehensive research tool, and no short version aimed specifically at clinical application has been published in the scientific literature. To utilize the aforementioned research findings in clinical practice, a pragmatic and convenient instrument with fewer items needs to be developed.

The objective of the study was threefold: 1) To develop a new instrument for identifying dysfunctional patients in a clinical setting, with adequate sensitivity, specificity, and receiver operating characteristics. 2) To assess the instrument's ability to reproduce the previously published effect estimates of $M C$, and 3 ) 
To test the sensitivity, specificity, and receiver operating characteristics in 3 other existing datasets to assess validity across populations.

\section{Method}

To address the first two objectives, data from a recently conducted randomized clinical trial (dataset 1 ) was utilized, and to answer the last objective, data from 3 other clinical trials described below (datasets 2 , 3 , and 4) were used.

Objective 1 (instrument development) was addressed using a cross-sectional analysis of the MPI-S data collected at baseline in dataset 1 .

Objective 2 (reproduction of effect) was answered using a longitudinal analysis of data from patients who completed the 12-month RCT comparing the intervention (MC) to the control group (dataset 1) with regards to the total number of days with activity limiting LBP for patients classified as dysfunctional at each level of the MAINTAIN instrument. These effect estimates along with the test statistics estimated in objective 1 were used to suggest clinical thresholds for a positive test.

Objective 3 (test of the instrument) was answered using a cross-sectional analysis of the MPI-S data collected at baseline in datasets 2,3 , and 4 , with the same procedure used in objective 1 , to explore the stability of the instrument's psychometric properties across populations.

\section{Study participants}

Dataset 1 was drawn from a randomized controlled trial with a 12-month follow-up period (19-22) investigating the effectiveness and cost-effectiveness of $M C$ in a population of patients with recurrent or persistent LBP from chiropractic primary care clinics in Sweden. The trial started in 2012 and was concluded in 2016 with a total of 249 patients completing the 12-month study period with a complete MPI-S dataset.

Dataset 2 was drawn from a large intervention study entitled "Work and Health in the Processing and Engineering Industries" conducted between 2000 and 2003 at four companies in Sweden $(48,49)$. Individuals classified as having a high risk of developing chronic disabling neck pain (NP) and/or LBP and long-term sick leave with a comprehensive risk assessment tool were selected for this study.

The third and fourth datasets came from a large intervention study entitled "Health-economic Evaluation and Rehabilitation (HUR)" conducted between 1994 and 1997 to evaluate multidisciplinary rehabilitation interventions with regards to their effect on sick leave, health-related quality of life, and costeffectiveness. Dataset 3 was drawn from an observational outcome study nested within the larger HUR project consisting of subjects suffering from neck and low back pain with intermittent sickness absence $(42,50,51)$. Dataset 4 was extracted from a randomized controlled trial also nested within the larger HUR project consisting of subjects with ongoing sickness absence as a result of NP and LBP $(52,53)$. Data 
were collected as part of the baseline assessment at the initial visit to the clinics. The research projects have been described in detail elsewhere (50-53).

The second, third, and fourth datasets were chosen as they represent a broad selection of different populations with LBP with different severity and consequences with regard to the patient's condition, i.e., working, on intermittent sick leave, or ongoing sick leave. These 4 populations have been compared with regards to psychological characteristics in a previous publication (54).

\section{Measures and outcomes}

Based on 8 items from the original MPI-S instrument suggested by Turk and Melzac (55), an instrument was developed and different scoring algorithms were explored. During the process, 2 additional items were added to improve discrimination between dysfunctional and interpersonally distressed individuals. A final scoring algorithm was decided by considering an optimal compromise between discriminant ability and ease of use in a clinical setting.

Outcomes of the study were test characteristics (Sensitivity, Specificity, Positive Predictive Value (PPV), Negative Predictive Value (NPV), Receiver Operating Characteristics (ROC), and Youden's index) of the MAINTAIN instrument's ability to classify dysfunctional subjects according to the original classification algorithm used by the original MPI-S instrument (gold standard).

\section{Data analysis}

Instrument development: Based on 10 items from the original MPI-S questionnaire, 5 dimensions of the patients' pain experience were recorded: pain severity (Q1 + Q7), interference (Q4 + Q8), life control (Q15 + $\mathrm{Q} 18)$, affective distress (Q22 + Q20) and support (Q5+Q14). Tentatively, a dysfunctional patient would score high on pain severity, interference, affective distress, support, and low on life control. A summary score was created based on this assumption, adding together the items from the high dimensions, and subtracting the score from the expected low dimension. The following scoring algorithm was created to generate a summative MAINTAIN Score: Q1 + Q7 + Q4 + Q8 - Q15 - Q18 + Q22 + Q20 + Q5 + Q14, ranging from -12 to 48 . Based on this scoring algorithm, each level of the MAINTAIN Score was analysed as a possible threshold for a dysfunctional classification and compared to the original MPI-S subgroup classification. A positive test would signify a dysfunctional profile and a negative test would signify either an interpersonally distressed or an adaptive coper profile. For each level of the MAINTAIN Score, the proportion of individuals for each subgroup who had a positive test was calculated. This data was graphically depicted to illustrate the relative frequency (\%) of true and false positive tests within each subgroup. In addition, a ROC was generated to estimate the AUC to further report the diagnostic ability of the instrument.

Reproduction of effect estimates: The difference in the total number of days with pain between the control and intervention groups in sample 1 was estimated for each MAINTAIN score using a general linear regression model to understand how well the instrument could reproduce the relevant subgroup previously shown to respond well to MC. Using the data on clinical outcomes along with the data on 
instrument performance from the development stage, two specific thresholds were identified to classify patients into: low, moderate, and high probability of MC being effective.

Assessing the stability of the instrument: Finally, the MAINTAIN instrument was tested in 3 separate data materials of patient populations who were experiencing pain resulting in different degrees of activity limitation collected in different settings to establish whether the test statistics (Sensitivity, Specificity, PPV, NPV, ROC and Youden's index) were valid (criterion) and reliable across populations.

\section{Ethical considerations}

The project was approved by the Swedish Ethical Review Authority (Ref.: 2019-04505). No new data was collected. Data had been handled and stored in accordance with the tenets of the World Medical Association's Declaration of Helsinki, and all the subjects had previously signed informed consent forms and agreed to participate in each of the four studies.

\section{Results}

In Table 1, the demographic data of samples 1-4 is displayed. 
Table 1

patient demographics of the 4 study samples.

\begin{tabular}{|c|c|c|c|c|}
\hline Variable & Sample 1 & Sample 2 & Sample 3 & Sample 4 \\
\hline $\begin{array}{l}\text { Description of } \\
\text { recruitment sites and } \\
\text { sample } \\
\text { characteristics }\end{array}$ & $\begin{array}{l}\text { Primary care } \\
\text { "Chiropractic", } \\
\text { not sick-listed }\end{array}$ & $\begin{array}{l}\text { Primary care, sick- } \\
\text { listed with a high } \\
\text { risk of chronicity }\end{array}$ & $\begin{array}{l}\text { Secondary } \\
\text { care, recurrent } \\
\text { sick leave }\end{array}$ & $\begin{array}{l}\text { Secondary } \\
\text { care, ongoing } \\
\text { sick leave }\end{array}$ \\
\hline $\mathrm{n}$ & 249 & 128 & 251 & 184 \\
\hline Female, n (\%) & $154(61.8)$ & $14(10.9)$ & $140(55.8)$ & $101(54.9)$ \\
\hline Age, Mean (SD) & $43.4(11.9)$ & $41.5(9.6)$ & $42.3(9.3)$ & $43.6(10.3)$ \\
\hline $\begin{array}{l}\text { Pain Severity }{ }^{1} \text {, Mean } \\
\text { (SD) }\end{array}$ & $3.3(1.1)$ & $2.8(1.3)$ & $3.5(1.1)$ & $3.7(1.1)$ \\
\hline $\begin{array}{l}\text { Interference }{ }^{1} \text {, Mean } \\
\text { (SD) }\end{array}$ & $2.8(1.3)$ & $2.5(1.3)$ & $3.8(1.1)$ & $3.8(1.0)$ \\
\hline $\begin{array}{l}\text { Life Control }{ }^{1}, \text { Mean } \\
\text { (SD) }\end{array}$ & $3.5(1.1)$ & $3.8(1.1)$ & $3.2(1.1)$ & $3.1(1.2)$ \\
\hline $\begin{array}{l}\text { Affective Distress }{ }^{1} \text {, } \\
\text { Mean (SD) }\end{array}$ & $2.7(1.3)$ & $2.3(1.4)$ & $2.6(1.6)$ & $2.8(1.5)$ \\
\hline Support ${ }^{1}$, Mean (SD) & $4.1(1.6)$ & $4.2(1.6)$ & $4.6(1.5)$ & $4.8(1.6)$ \\
\hline $\begin{array}{l}\text { Punishing } \\
\text { Responses }{ }^{1} \text {, Mean } \\
\text { (SD) }\end{array}$ & $1.1(1.3)$ & $1.3(1.3)$ & $1.1(1.2)$ & $1.0(1.2)$ \\
\hline $\begin{array}{l}\text { Solicitous } \\
\text { Responses }{ }^{1} \text {, Mean } \\
\text { (SD) }\end{array}$ & $2.7(1.4)$ & $2.9(1.4)$ & $3.2(1.4)$ & $3.1(1.4)$ \\
\hline $\begin{array}{l}\text { Distracting } \\
\text { responses }^{1} \text {, Mean } \\
(\mathrm{SD})\end{array}$ & $2.8(1.4)$ & $3.0(1.6)$ & $3.1(1.6)$ & $3.2(1.6)$ \\
\hline $\begin{array}{l}\text { MPI-Subgroup, \% } \\
\text { (AC/ID/DYS) }\end{array}$ & $36.5 / 24.9 / 38.6$ & $48.4 / 25.8 / 25.8$ & $24.9 / 24.5 / 50.6$ & $21.2 / 25.5 / 53.3$ \\
\hline $\begin{array}{l}\text { MAINTAIN Score, -12- } \\
48 \text {, Mean (SD) }\end{array}$ & $20.0(8.4)$ & $16.5(9.7)$ & $23.0(8.9)$ & $23.5(8.1)$ \\
\hline
\end{tabular}

\section{Objective 1, Instrument development}


Based on data from sample 1, three different MAINTAIN thresholds were selected. First, a lower threshold was chosen where most dysfunctional individuals can be identified with a reasonable number of false positive tests with particular attention to AC individuals. The threshold of 18 resulted in a reasonable trade-off where $95.8 \%$ of all DYS individuals were correctly classified and only $17.4 \%$ of AC individuals were falsely classified as DYS. At this level, $62.9 \%$ of ID individuals were falsely classified as DYS. At a score of 18 or higher, 146 out of the 249 patients were classified as dysfunctional with $95.8 \%$ sensitivity and $64.3 \%$ specificity. Second, an upper threshold where most of the AC individuals have been excluded at the expense of a reasonable number of false negative tests with respect to the DYS individuals was selected. At the higher threshold of $22,81.1 \%$ of all DYS individuals were correctly classified and only $4.3 \%$ of AC individuals were falsely classified as DYS. At this level, $45.2 \%$ of all ID individuals were falsely classified as DYS. At a score of 22 or higher, 109 were classified as dysfunctional with $81.1 \%$ sensitivity and $79.2 \%$ specificity. Third, a threshold with the optimum cut-off point where equal weight is given to false positive and false negative values (highest value on the Youden's index, 0.70 ) was identified at a MAINTAIN score of 20. At this level, 133 individuals were classified as dysfunctional with $90.5 \%$ sensitivity and $69.5 \%$ specificity. The AUC was estimated to 0.87 (95\% Cl: $0.83,0.92 ; p<0.001)$. The test statistics for each of these scores of the MAINTAIN instrument in sample 1 are reported in Table 2 . In Fig. 1, the proportion of individuals classified with the original MPI-S instrument with a positive test per level of the MAINTAIN instrument is reported. The MAINTAIN instrument is presented in Additional file 1. 
Table 2

Test statistics in sample 1 (dysfunctional patients classified by original MPI-S instrument, $n=96$ )

\section{Sample $1(n=249)$}

\begin{tabular}{|c|c|c|c|c|c|c|}
\hline MS & n (DYS) & Sensitivity (\%) & Specificity (\%) & PPV (\%) & NPV (\%) & Youden's index \\
\hline 8 & 229 & 100.0 & 13.0 & 41.5 & 100.0 & 0.110 \\
\hline 9 & 224 & 100.0 & 16.2 & 42.4 & 100.0 & 0.130 \\
\hline 10 & 220 & 100.0 & 18.8 & 43.2 & 100.0 & 0.162 \\
\hline 11 & 218 & 100.0 & 20.1 & 43.6 & 100.0 & 0.188 \\
\hline 12 & 205 & 100.0 & 28.6 & 46.3 & 100.0 & 0.201 \\
\hline 13 & 201 & 100.0 & 31.2 & 47.3 & 100.0 & 0.286 \\
\hline 14 & 193 & 100.0 & 36.4 & 49.2 & 100.0 & 0.312 \\
\hline 15 & 180 & 98.9 & 44.2 & 52.2 & 98.6 & 0.364 \\
\hline 16 & 173 & 98.9 & 48.7 & 54.3 & 98.7 & 0.431 \\
\hline 17 & 159 & 96.8 & 56.5 & 57.9 & 96.7 & 0.476 \\
\hline 18 & 146 & 95.8 & 64.3 & 62.3 & 96.1 & 0.533 \\
\hline 19 & 140 & 94.7 & 67.5 & 64.3 & 95.4 & 0.601 \\
\hline 20 & 133 & 90.5 & 69.5 & 64.7 & 92.2 & 0.623 \\
\hline 21 & 122 & 86.3 & 74.0 & 67.2 & 89.8 & 0.600 \\
\hline 22 & 109 & 81.1 & 79.2 & 70.6 & 87.1 & 0.603 \\
\hline 23 & 103 & 75.8 & 79.9 & 69.9 & 84.2 & 0.603 \\
\hline 24 & 92 & 70.5 & 83.8 & 72.8 & 82.2 & 0.557 \\
\hline 25 & 84 & 63.2 & 84.4 & 71.4 & 78.8 & 0.543 \\
\hline 26 & 74 & 60.0 & 89.0 & 77.0 & 78.3 & 0.476 \\
\hline 27 & 67 & 55.8 & 90.9 & 79.1 & 76.9 & 0.490 \\
\hline
\end{tabular}

\section{Objective 2, reproduction of effect:}

Data on clinical outcomes from sample 1, number of days with activity limiting pain (primary outcome), was used to estimate the effect of the intervention (difference between $\mathrm{MC}$ and control) for patients 
classified as Dysfunctional at each score of the MAINTAIN instrument. A positive trend was observed, with a higher effect of MC with a higher MAINTAIN score in a dose-response-like relationship. Patients in the MC-group classified as dysfunctional at a score of 18 or higher, experienced on average 24.4 (95\% Cl: $-0.3,49.1)$ fewer days with activity limiting pain compared to dysfunctional patients in the control group, whereas those classified at a score of 22 or higher reported on average $34.5(95 \% \mathrm{Cl}: 5.2,63.7)$ fewer days with activity limiting pain. Effect estimates for each score of the MAINTAIN instrument are reported in Table 3. 
Table 3

Effect of maintenance care with a dysfunctional classification for each of the scores 8-27 on the MAINTAIN instrument.

\begin{tabular}{|c|c|c|c|}
\hline MS & n (DYS) & $\begin{array}{l}\text { Difference in total number of days with activity limiting LBP, } \\
\text { MC- Control }(95 \% \mathrm{Cl}) \text {, sample } 1(\mathrm{n}=249)\end{array}$ & p-value \\
\hline 8 & 229 & $-13.6(-32.1,5.0)$ & 0.152 \\
\hline 9 & 224 & $-13.3(-32.2,5.7)$ & 0.169 \\
\hline 10 & 220 & $-16.0(-34.9,3.0)$ & 0.099 \\
\hline 11 & 218 & $-16.6(-35.8,2.5)$ & 0.088 \\
\hline 12 & 205 & $-17.7(-37.7,2.4)$ & 0.083 \\
\hline 13 & 201 & $-16.3(-36.6,4.0)$ & 0.115 \\
\hline 14 & 193 & $-19.7(-40.5,1.1)$ & 0.063 \\
\hline 15 & 180 & $-19.6(-41.3,2.1)$ & 0.076 \\
\hline 16 & 173 & $-22.2(-44.5,0.0)$ & 0.050 \\
\hline 17 & 159 & $-22.9(-46.3,0.5)$ & 0.055 \\
\hline 18 & 146 & $-24.4(-49.1,0.3)$ & 0.053 \\
\hline 19 & 140 & $-28.9(-53.6,-4.2)$ & 0.022 \\
\hline 20 & 133 & $-31.5(-57.2,-5.8)$ & 0.017 \\
\hline 21 & 122 & $-32.6(-59.8,-5.4)$ & 0.019 \\
\hline 22 & 109 & $-34.5(-63.7,-5.2)$ & 0.021 \\
\hline 23 & 103 & $-34.1(-64.6,-3.6)$ & 0.029 \\
\hline 24 & 92 & $-29.9(-61.6,1.7)$ & 0.064 \\
\hline 25 & 84 & $-27.2(-60.0,5.6)$ & 0.103 \\
\hline 26 & 74 & $-30.0(-66.2,6.2)$ & 0.103 \\
\hline 27 & 67 & $-25.1(-64.0,13.9)$ & 0.204 \\
\hline
\end{tabular}

\section{Objective 3, test of the instrument:}

In sample 2, at a score of 18 or higher, 61 out of the 128 patients were classified as Dysfunctional with $93.9 \%$ sensitivity and $68.4 \%$ specificity. At a score of 22 or higher, 41 were classified as dysfunctional 
with $84.8 \%$ sensitivity and $86.3 \%$ specificity. In sample 3, at a score of 18 or higher, 183 out of the 251 patients were classified as Dysfunctional with $96.1 \%$ sensitivity and $50.8 \%$ specificity. At a score of 22 or higher, 148 were classified as Dysfunctional with $85.8 \%$ sensitivity and $68.5 \%$ specificity. In sample 4 , at a score of 18 or higher, 142 out of the 184 patients were classified as dysfunctional with $95.9 \%$ sensitivity and $44.2 \%$ specificity. At a score of 22 or higher 108 were classified as Dysfunctional with 80.6 $\%$ sensitivity and $66.3 \%$ specificity.

The test showed acceptable to good performance (ROC characteristics) in all samples with high AUC estimates that were statistically significant. When considering the optimum cut-off point where equal weight is given to false positive and false negative values (highest value on the Youden's index) the thresholds differ somewhat in samples 1 compared to samples 2 and 3 . In sample 4, the highest index (0.451) can be found at a MAINTAIN score of 20 resembling the data from sample 1 . Whereas the highest index in samples 2 and 3 can be found at a MAINTAIN score of $25(0.712)$ and $27(0.590)$, respectively.

The test statistics for each score of the MAINTAIN instrument in samples 2 to 4 are reported in Tables 46. The AUC for samples 1 to 4 is reported in Table 7. 
Table 4

Test statistics in sample 2

\section{Sample $2(n=128)$}

\begin{tabular}{|llllll|}
\hline MS & Sensitivity (\%) & Specificity (\%) & PPV (\%) & NPV (\%) & Youden's index \\
\hline 8 & 100.0 & 22.1 & 30.8 & 100.0 & 0.158 \\
\hline 9 & 100.0 & 27.4 & 32.4 & 100.0 & 0.179 \\
\hline 10 & 100.0 & 31.6 & 33.7 & 100.0 & 0.200 \\
\hline 11 & 100.0 & 37.9 & 35.9 & 100.0 & 0.221 \\
\hline 12 & 100.0 & 41.1 & 37.1 & 100.0 & 0.274 \\
\hline 13 & 97.0 & 48.4 & 39.5 & 97.9 & 0.316 \\
\hline 14 & 93.9 & 53.7 & 41.3 & 96.2 & 0.379 \\
\hline 15 & 93.9 & 56.8 & 43.1 & 96.4 & 0.411 \\
\hline 16 & 93.9 & 61.1 & 45.6 & 96.7 & 0.454 \\
\hline 17 & 93.9 & 67.4 & 50.0 & 97.0 & 0.476 \\
\hline 18 & 93.9 & 68.4 & 50.8 & 97.0 & 0.508 \\
\hline 19 & 90.9 & 71.6 & 52.6 & 95.8 & 0.550 \\
\hline 20 & 90.9 & 78.9 & 60.0 & 96.2 & 0.613 \\
\hline 21 & 84.8 & 82.1 & 62.2 & 94.0 & 0.624 \\
\hline 22 & 84.8 & 86.3 & 68.3 & 94.3 & 0.625 \\
\hline 23 & 78.8 & 88.4 & 70.3 & 92.3 & 0.699 \\
\hline 24 & 69.7 & 89.5 & 69.7 & 89.5 & 0.670 \\
\hline 25 & 60.6 & 90.5 & 69.0 & 86.9 & 0.712 \\
\hline 26 & 54.5 & 94.7 & 85.6 & 0.672 \\
\hline 27 & 48.5 & 76.0 & 84.1 & 0.592 \\
\hline MS, MAINTAIN Score; $\mathrm{n}(\mathrm{DYS})$ & number of individuals classified as dysfunctional by the MAINTAIN \\
instrument at that threshold; PPV, Positive Predictive Value; NPV, Negative Predictive Value
\end{tabular}


Table 5

Test statistics in sample 3

\begin{tabular}{|c|c|c|c|c|c|}
\hline \multicolumn{6}{|c|}{ Sample $3(n=251)$} \\
\hline MS & Sensitivity (\%) & Specificity (\%) & PPV (\%) & NPV (\%) & Youden's index \\
\hline 8 & 100.0 & 5.6 & 52.0 & 100.0 & 0.040 \\
\hline 9 & 100.0 & 6.5 & 52.3 & 100.0 & 0.051 \\
\hline 10 & 100.0 & 10.5 & 53.4 & 100.0 & 0.070 \\
\hline 11 & 100.0 & 13.7 & 54.3 & 100.0 & 0.083 \\
\hline 12 & 100.0 & 18.5 & 55.7 & 100.0 & 0.098 \\
\hline 13 & 100.0 & 21.8 & 56.7 & 100.0 & 0.115 \\
\hline 14 & 100.0 & 27.4 & 58.5 & 100.0 & 0.130 \\
\hline 15 & 99.2 & 34.7 & 60.9 & 97.7 & 0.161 \\
\hline 16 & 98.4 & 40.3 & 62.8 & 96.2 & 0.193 \\
\hline 17 & 96.9 & 47.6 & 65.4 & 93.7 & 0.231 \\
\hline 18 & 96.1 & 50.8 & 66.7 & 92.6 & 0.261 \\
\hline 19 & 95.3 & 56.5 & 69.1 & 92.1 & 0.296 \\
\hline 20 & 92.1 & 62.1 & 71.3 & 88.5 & 0.345 \\
\hline 21 & 89.0 & 64.5 & 72.0 & 85.1 & 0.397 \\
\hline 22 & 85.8 & 68.5 & 73.6 & 82.5 & 0.431 \\
\hline 23 & 82.7 & 71.0 & 74.5 & 80.0 & 0.486 \\
\hline 24 & 76.4 & 75.8 & 76.4 & 75.8 & 0.531 \\
\hline 25 & 69.3 & 78.2 & 76.5 & 71.3 & 0.560 \\
\hline 26 & 63.0 & 84.7 & 80.8 & 69.1 & 0.577 \\
\hline 27 & 55.9 & 86.3 & 80.7 & 65.6 & 0.590 \\
\hline
\end{tabular}


Table 6

Test statistics in sample 4

\begin{tabular}{|c|c|c|c|c|c|}
\hline \multicolumn{6}{|c|}{ Sample $4(n=184)$} \\
\hline MS & Sensitivity (\%) & Specificity (\%) & PPV (\%) & NPV (\%) & Youden's index \\
\hline 8 & 100.0 & 5.8 & 54.7 & 100.0 & 0.198 \\
\hline 9 & 100.0 & 7.0 & 55.1 & 100.0 & 0.244 \\
\hline 10 & 100.0 & 11.6 & 56.3 & 100.0 & 0.267 \\
\hline 11 & 100.0 & 14.0 & 57.0 & 100.0 & 0.269 \\
\hline 12 & 100.0 & 16.3 & 57.6 & 100.0 & 0.339 \\
\hline 13 & 100.0 & 19.8 & 58.7 & 100.0 & 0.401 \\
\hline 14 & 100.0 & 24.4 & 60.1 & 100.0 & 0.427 \\
\hline 15 & 100.0 & 26.7 & 60.9 & 100.0 & 0.401 \\
\hline 16 & 99.0 & 27.9 & 61.0 & 96.0 & 0.428 \\
\hline 17 & 99.0 & 34.9 & 63.4 & 96.8 & 0.469 \\
\hline 18 & 95.9 & 44.2 & 66.2 & 90.5 & 0.462 \\
\hline 19 & 93.9 & 48.8 & 67.6 & 87.5 & 0.443 \\
\hline 20 & 87.8 & 52.3 & 67.7 & 78.9 & 0.451 \\
\hline 21 & 84.7 & 58.1 & 69.7 & 76.9 & 0.415 \\
\hline 22 & 80.6 & 66.3 & 73.1 & 75.0 & 0.334 \\
\hline 23 & 77.6 & 68.6 & 73.8 & 72.8 & 0.317 \\
\hline 24 & 74.5 & 69.8 & 73.7 & 70.6 & 0.258 \\
\hline 25 & 68.4 & 76.7 & 77.0 & 68.0 & 0.210 \\
\hline 26 & 61.2 & 80.2 & 77.9 & 64.5 & 0.182 \\
\hline 27 & 52.0 & 81.4 & 76.1 & 59.8 & 0.146 \\
\hline
\end{tabular}


Table 7

Area Under the ROC Curve statistics for MAINTAIN instrument in samples 1-4.

\begin{tabular}{|llll|}
\hline Sample & $\mathbf{n}$ & AUC $(95 \% \mathrm{Cl})$ & p-value \\
\hline 1 & 249 & $0.875(0.833,0.917)$ & $<0.001$ \\
2 & 128 & $0.902(0.846,0.958)$ & $<0.001$ \\
3 & 251 & $0.844(0.795,0.892)$ & $<0.001$ \\
4 & 184 & $0.791(0.724,0.857)$ & $<0.001$ \\
\hline
\end{tabular}

\section{Discussion}

To our knowledge, this is the first study to develop and psychometrically assess a short version of MPI-S with the purpose of identifying dysfunctional subjects suitable for MC in a clinical setting, see Additional file 1. The MAINTAIN instrument has the potential of being an effective clinical decision aid allowing clinicians to acquire a relevant score of psychological distress in a short period with a low administrative load. The instrument has displayed an acceptable trade-off between sensitivity and specificity across contexts and populations and can be used with different thresholds depending on the rate of false positive tests that can be accepted in the clinical encounter. The instrument has exhibited high ROC statistics with scores ranging between 0.79 and 0.90 , thus, suggesting acceptable to excellent discriminant ability (56). Rather than using a single threshold with a dichotomous outcome we recommend 3 ranges to illustrate that the instrument represents a scale where the likelihood of a good outcome from MC changes in a dose-response-like relationship. We suggest clinicians use the instrument as one parameter in a broader assessment strategy where previous pain trajectory, initial treatment effectiveness, ability to perform active strategies, and patient preferences should be considered.

In the present work, samples collected from patient populations experiencing pain which resulted in different degrees of activity limitation collected in different settings allowed for a robust evaluation and introspection of the instrument's performance. Also, by verifying the stratification procedure using clinical outcomes, the clinical usefulness of the instrument is assessed. We considered the data from this trial to be robust and trustworthy, and the MAINTAIN instrument adds a valuable contribution by informing clinical decision-making and deepening the understanding of the psychological consequences of the patient's condition. The advantage of a short instrument with a simple scoring algorithm is the possibility to employ it in the clinical encounter and being able to score while the patient is present. This allows the clinician to promptly get an overview of the psychological dimensions of the patient's pain experience as well as an opportunity to dwell into each of the 5 dimensions, pain severity, interference, life control, affective distress, and support, with probing follow-up questions. In addition to deepening the understanding of the patient's pain condition, this information can be used to establish treatment goals or adapt the intervention to better align with the patients' needs and preferences. 
Efforts have been made to find robust solutions to tailor treatments to specific subgroups of patients with recurrent or persistent LBP. However, none of the available instruments have been able to improve outcomes among chiropractic patients by identifying patients suitable for a stratified care pathway. Previous research has investigated the predictive ability of the Keele STarT Back Tool when implemented in chiropractic practice and has found its prognostic ability to be limited among patients with shorter LBP episodes, which is a common presentation in chiropractic patients (57). Patients included in sample 1 , where the effect and cost-effectiveness of MC were investigated, were included only if they reported recurrent or persistent pain, and the long-term effect over 12 months was studied. This may explain the good to excellent discriminant ability of the MAINTAIN instrument in contrast to the research done on the Keele STarT Back Tool. Thus, psychometric properties, such as validity, may not pertain to an instrument as such; rather, they are a feature of the construal of the results generated from a contextual study.

Future research should further investigate the use of the MAINTAIN instrument in a clinical setting in an implementation trial by comparing a stratified MC approach resulting from using the MAINTAIN instrument and comparing it with standard care, exercise interventions, and digital consultations. Also, it would be interesting to contrast and compare the thresholds to the Keele STarT Back Tool and the Örebro Musculoskeletal Screening Pain Questionnaire (ÖMSPQ) to further explore the construct validity and usefulness of the MAINTAIN tool to populations with recurrent or persistent LBP. Such data could shed light on the possibility of using these instruments as decision-making aids for identifying patients suitable for MC.

\section{Conclusion}

The MAINTAIN is a short instrument with a simple scoring algorithm that has an acceptable performance with regard to selecting dysfunctional patients in a clinical setting. By using 2 thresholds, patients can be categorized into "low probability", "moderate probability" and "high probability" of having a good outcome from maintenance care for LBP. The discriminant ability is similar and acceptable across populations with minor differences in optimal thresholds for identifying dysfunctional individuals. Implementing the MAINTAIN instrument has the potential of improving outcomes by identifying dysfunctional high-risk patients early in the clinical course and stratifying them to appropriate interventions with a higher chance of treatment success.

\section{List Of Abbreviations}

AC; Adaptive Coper

DYS; Dysfunctional

ID; Interpersonally Distressed

LBP; Low Back Pain

Page 18/24 
MC; Chiropractic Maintenance Care

MPI-S; Swedish version of West Haven-Yale Multidimensional Pain Inventory

\section{Declarations}

\section{Ethics approval and consent to participate}

The trial was approved by Swedish Ethical Review Authority (ref: 2019-04505). All participants provided a signed informed consent in order to participate.

\section{Consent for publication}

Not applicable

\section{Availability of data and materials}

Data cannot be shared publicly for legal reasons. Data will be available upon request after permission is granted by the Swedish Ethical Review Authority: kansli@stockholm.epn.se. Inquiries for data access should first be sent to irene.jensen@ki.se, who will then contact the ethics board for permission to openly share the data.

\section{Competing interests}

The lead author Andreas Eklund is a paid employee of a commercial company who works part-time (40\%) as a clinician at Hälsan Östertälje Praktikertjänst AB, Södertälje, Sweden. The affiliation has not influenced the study design, data collection, and analysis, decision to publish, or preparation of the manuscript. There are no patents, products in development, or marketed products associated with this research to declare. IA is co-editor-in-chief and on the editorial board of BMC Chiropractic and Manual Therapies. The authors declare that they have no competing interests.

\section{Funding}

This study was funded by the European Center for Chiropractic Research Excellence (ECCRE), https://nikkb.dk/eccre/ (grant number 25-2018-SE/AE) 75\% and Karolinska Institutet 25\%. The funder provided support in the form of salaries for authors AE but had no role in the study design, data collection, and analysis, decision to publish, or preparation of the manuscript.

\section{Authors' contributions}

Andreas Eklund is the corresponding author and is involved in conceptualization, data curation, formal analysis, funding acquisition, investigation, methodological development, project administration, validation, visualization, writing the original draft, reviewing and editing the final manuscript. 
Per J. Palmgren, Ulf Jakobsson, and Iben Axén were involved in conceptualization, methodological development, reviewing, and editing the final manuscript.

\section{Acknowledgements}

The authors wish to acknowledge the dedicated clinicians of the Swedish Chiropractic Association (LKR) who collected the data in this trial and made a major contribution. We also wish to acknowledge the trust that the European Centre for Research Excellence (ECCRE) and the chiropractic profession have shown in our research group by their support and funding.

\section{References}

1. Murray CJ, Lopez AD. Measuring the global burden of disease. N Engl J Med. 2013;369(5):448-57.

2. Hoy D, March L, Brooks P, Blyth F, Woolf A, Bain C, et al. The global burden of low back pain: estimates from the Global Burden of Disease 2010 study. Ann Rheum Dis. 2014;73(6):968-74.

3. Steffens D, Maher CG, Pereira LS, Stevens ML, Oliveira VC, Chapple M, et al. Prevention of Low Back Pain: A Systematic Review and Meta-analysis. JAMA Intern Med. 2016;176(2):199-208.

4. Breen AC. Chiropractors and the treatment of back pain. Rheumatol Rehabil. 1977;16(1):46-53.

5. Mitchell M. Maintenance care. Some considerations. The ACA Journal of Chiropractic. 1980(17):535.

6. Rupert RL. A survey of practice patterns and the health promotion and prevention attitudes of US chiropractors. Maintenance care: part I. J Manipulative Physiol Ther. 2000;23(1):1-9.

7. Rupert RL, Manello D, Sandefur R. Maintenance care: health promotion services administered to US chiropractic patients aged 65 and older, part II. J Manipulative Physiol Ther. 2000;23(1):10-9.

8. Axen I, Rosenbaum A, Eklund A, Halasz L, Jorgensen K, Lovgren PW, et al. The Nordic maintenance care program - case management of chiropractic patients with low back pain: a survey of Swedish chiropractors. Chiropr Osteopat. 2008;16:6.

9. Leboeuf-Yde C, Hestbaek L. Maintenance care in chiropractic-what do we know? Chiropr Osteopat. 2008;16:3.

10. Axen I, Jensen IB, Eklund A, Halasz L, Jorgensen K, Lange F, et al. The Nordic Maintenance Care Program: when do chiropractors recommend secondary and tertiary preventive care for low back pain? Chiropr Osteopat. 2009;17:1.

11. Malmqvist S, Leboeuf-Yde $C$. The Nordic maintenance care program: case management of chiropractic patients with low back pain-defining the patients suitable for various management strategies. Chiropr Osteopat. 2009;17:7.

12. Moller LT, Hansen M, Leboeuf-Yde C. The Nordic Maintenance Care Program--an interview study on the use of maintenance care in a selected group of Danish chiropractors. Chiropr Osteopat. 2009;17:5. 
13. Hansen SF, Laursen ALS, Jensen TS, Leboeuf-Yde C, L H. The Nordic maintenance care program: what are the indications for maintenance care in patients with low back pain? A survey of the members of the Danish Chiropractors' Association. Chiropr Osteopat. 2010;18:25.

14. Sandnes KF, Bjørnstad C, Leboeuf-Yde C, Hestbaek L. The Nordic Maintenance Care Program - Time intervals between treatments of patients with low back pain: how close and who decides? Chiropractic \& Osteopathy. 2010;18(1):5.

15. Bringsli M, Berntzen A, Olsen DB, Leboeuf-Yde C, Hestbaek L. The Nordic Maintenance Care Program: Maintenance care - what happens during the consultation? Observations and patient questionnaires. Chiropr Man Therap. 2012;20(1):25.

16. Axen I, Bodin L. The Nordic maintenance care program: the clinical use of identified indications for preventive care. Chiropr Man Therap. 2013;21(1):10.

17. Myburgh C, Brandborg-Olsen D, Albert $H$, Hestbaek L. The Nordic maintenance care program: what is maintenance care? Interview based survey of Danish chiropractors. Chiropr Man Therap. 2013;21(1):27.

18. Iben A, Lise H, Charlotte LY. Chiropractic maintenance care - what's new? A systematic review of the literature. Chiropr Man Therap. 2019;27:63.

19. Eklund A, Axen I, Kongsted A, Lohela-Karlsson M, Leboeuf-Yde C, Jensen I. Prevention of low back pain: effect, cost-effectiveness, and cost-utility of maintenance care - study protocol for a randomized clinical trial. Trials. 2014;15(1):102.

20. Eklund A, Jensen I, Lohela-Karlsson M, Hagberg J, Leboeuf-Yde C, Kongsted A, et al. The Nordic Maintenance Care program: Effectiveness of chiropractic maintenance care versus symptom-guided treatment for recurrent and persistent low back pain-A pragmatic randomized controlled trial. PLoS One. 2018;13(9):e0203029.

21. Eklund A, Jensen I, Leboeuf-Yde C, Kongsted A, Jonsson M, Lovgren P, et al. The Nordic Maintenance Care Program: Does psychological profile modify the treatment effect of a preventive manual therapy intervention? A secondary analysis of a pragmatic randomized controlled trial. PLoS One. 2019;14(10):e0223349.

22. Eklund A, Hagberg J, Jensen I, Leboeuf-Yde C, Kongsted A, Lovgren P, et al. The Nordic maintenance care program: maintenance care reduces the number of days with pain in acute episodes and increases the length of pain-free periods for dysfunctional patients with recurrent and persistent low back pain-a secondary analysis of a pragmatic randomized controlled trial. Chiropractic \& Manual Therapies. 2020;28(1).

23. Hall AM, Ferreira PH, Maher CG, Latimer J, Ferreira ML. The Influence of the Therapist-Patient Relationship on Treatment Outcome in Physical Rehabilitation: A Systematic Review. Phys Ther. 2010;90(8):1099-110.

24. Melzack R, Wall PD. Pain mechanisms: a new theory. Science. 1965;150(3699):971-9.

25. Fordyce WE, Fowler RS, DeLateur B. An application of behavior modification technique to a problem of chronic pain. Behav Res Ther. 1968;6(1):105-7. 
26. Gatchel RJ TD. Psychosocial factors in pain: critical perspectives. New York: Guilford Press; 1999.

27. Burton AK, Tillotson KM, Main CJ, Hollis S. Psychosocial predictors of outcome in acute and subchronic low back trouble. Spine (Phila Pa 1976). 1995;20(6):722-8.

28. Hoogendoorn WE, van Poppel MN, Bongers PM, Koes BW, Bouter LM. Systematic review of psychosocial factors at work and private life as risk factors for back pain. Spine (Phila Pa 1976). 2000;25(16):2114-25.

29. Linton SJ, Boersma K. Early identification of patients at risk of developing a persistent back problem: the predictive validity of the Orebro Musculoskeletal Pain Questionnaire. Clin J Pain. 2003;19(2):80-6.

30. Linton SJ, Buer N, Vlaeyen J, Hellsing AL. Are fear-avoidance beliefs related to the inception of an episode of back pain? A prospective study. Psychol Health. 1999;14(6):1051-9.

31. Linton SJ, Hallden K. Can we screen for problematic back pain? A screening questionnaire for predicting outcome in acute and subacute back pain. Clin J Pain. 1998;14(3):209-15.

32. Linton SJ, Nicholas M, MacDonald S. Development of a short form of the Orebro Musculoskeletal Pain Screening Questionnaire. Spine. 2011;36.

33. Macfarlane GJ, Thomas E, Croft PR, Papageorgiou AC, Jayson MI, Silman AJ. Predictors of early improvement in low back pain amongst consulters to general practice: the influence of pre-morbid and episode-related factors. Pain. 1999;80(1-2):113-9.

34. Engel GL. The need for a new medical model: a challenge for biomedicine. Science. 1977;196(4286):129-36.

35. Engel GL. The clinical application of the biopsychosocial model. AJ Psychiatry. 1980;137(5):535-44.

36. Weiner BK. Spine update: the biopsychosocial model and spine care. Spine (Phila Pa 1976). 2008;33(2):219-23.

37. Kerns RD, Turk DC, Rudy TE. The West Haven-Yale Multidimensional Pain Inventory (WHYMPI). Pain. 1985;23(4):345-56.

38. Turk DC. The potential of treatment matching for subgroups of patients with chronic pain: lumping versus splitting. Clin J Pain. 2005;21(1):44-55; discussion 69-72.

39. Bergstrom G, Bodin L, Jensen IB, Linton SJ, Nygren AL. Long-term, non-specific spinal pain: reliable and valid subgroups of patients. Behav Res Ther. 2001;39(1):75-87.

40. Lousberg R, Schmidt AJ, Groenman NH, Vendrig L, Dijkman-Caes Cl. Validating the MPI-DLV using experience sampling data. J Behav Med. 1997;20(2):195-206.

41. Bergström C, Hagberg J, Bodin L, Jensen I, Bergström G. Using a psychosocial subgroup assignment to predict sickness absence in a working population with neck and back pain. BMC Musculoskeletal Disorders. 2011;12(1):81.

42. Bergstrom G, Bergstrom C, Hagberg J, Bodin L, Jensen I. A 7-year follow-up of multidisciplinary rehabilitation among chronic neck and back pain patients. Is sick leave outcome dependent on psychologically derived patient groups? Eur J Pain. 2010;14(4):426-33. 
43. Turk DC, Rudy TE. The robustness of an empirically derived taxonomy of chronic pain patients. Pain. 1990;43(1):27-35.

44. Turk DC. Strategies for classifying chronic orofacial pain patients. Anesth Prog. 1990;37(2-3):155-60.

45. Walter L, Brannon L. A cluster analysis of the multidimensional pain inventory. Headache. 1991;31(7):476-9.

46. Turk DC, Okifuji A, Sinclair JD, Starz TW. Pain, disability, and physical functioning in subgroups of patients with fibromyalgia. J Rheumatol. 1996;23(7):1255-62.

47. Turk DC, Sist TC, Okifuji A, Miner MF, Florio G, Harrison P, et al. Adaptation to metastatic cancer pain, regional/local cancer pain and non-cancer pain: role of psychological and behavioral factors. Pain. 1998;74(2-3):247-56.

48. Bergstrom G, Bjorklund C, Fried I, Lisspers J, Nathell L, Hermansson U, et al. A comprehensive workplace intervention and its outcome with regard to lifestyle, health and sick leave: the AHA study. Work. 2008;31(2):167-80.

49. Bergstrom C, Hagberg J, Bodin L, Jensen I, Bergstrom G. Using a psychosocial subgroup assignment to predict sickness absence in a working population with neck and back pain. BMC Musculoskelet Disord. 2011;12:81.

50. Jensen IB, G. Nygren, Å. Ljungquist, T. . Rehabilitation of patients suffering from spinal pain (Rehabillitering av patienter med smärttillstånd i ryggkotpelaren). Section for Personal Injury Prevention, Karolinska Institute, Stockholm; 1999.

51. Jensen IB, Busch H, Bodin L, Hagberg J, Nygren A, Bergstrom G. Cost effectiveness of two rehabilitation programmes for neck and back pain patients: A seven year follow-up. Pain. 2009;142(3):202-8.

52. Jensen IB, Bergstrom G, Ljungquist T, Bodin L, Nygren AL. A randomized controlled component analysis of a behavioral medicine rehabilitation program for chronic spinal pain: are the effects dependent on gender? Pain. 2001;91(1-2):65-78.

53. Busch H, Bodin L, Bergstrom G, Jensen IB. Patterns of sickness absence a decade after pain-related multidisciplinary rehabilitation. Pain. 2011;152(8):1727-33.

54. Eklund A, Bergstrom G, Bodin L, Axen I. Psychological and behavioral differences between low back pain populations: a comparative analysis of chiropractic, primary and secondary care patients. BMC Musculoskelet Disord. 2015;16(1):306.

55. Turk DC, Melzack R. Handbook of pain assessment. 1. ed. ed. New York: Guilford Press; 1992.

56. Obuchowski NA, Bullen JA. Receiver operating characteristic (ROC) curves: review of methods with applications in diagnostic medicine. Phys Med Biol. 2018;63(7):07TR1.

57. Khan Y. The STarT back tool in chiropractic practice: a narrative review. Chiropr Man Therap. 2017;25:11.

\section{Figures}




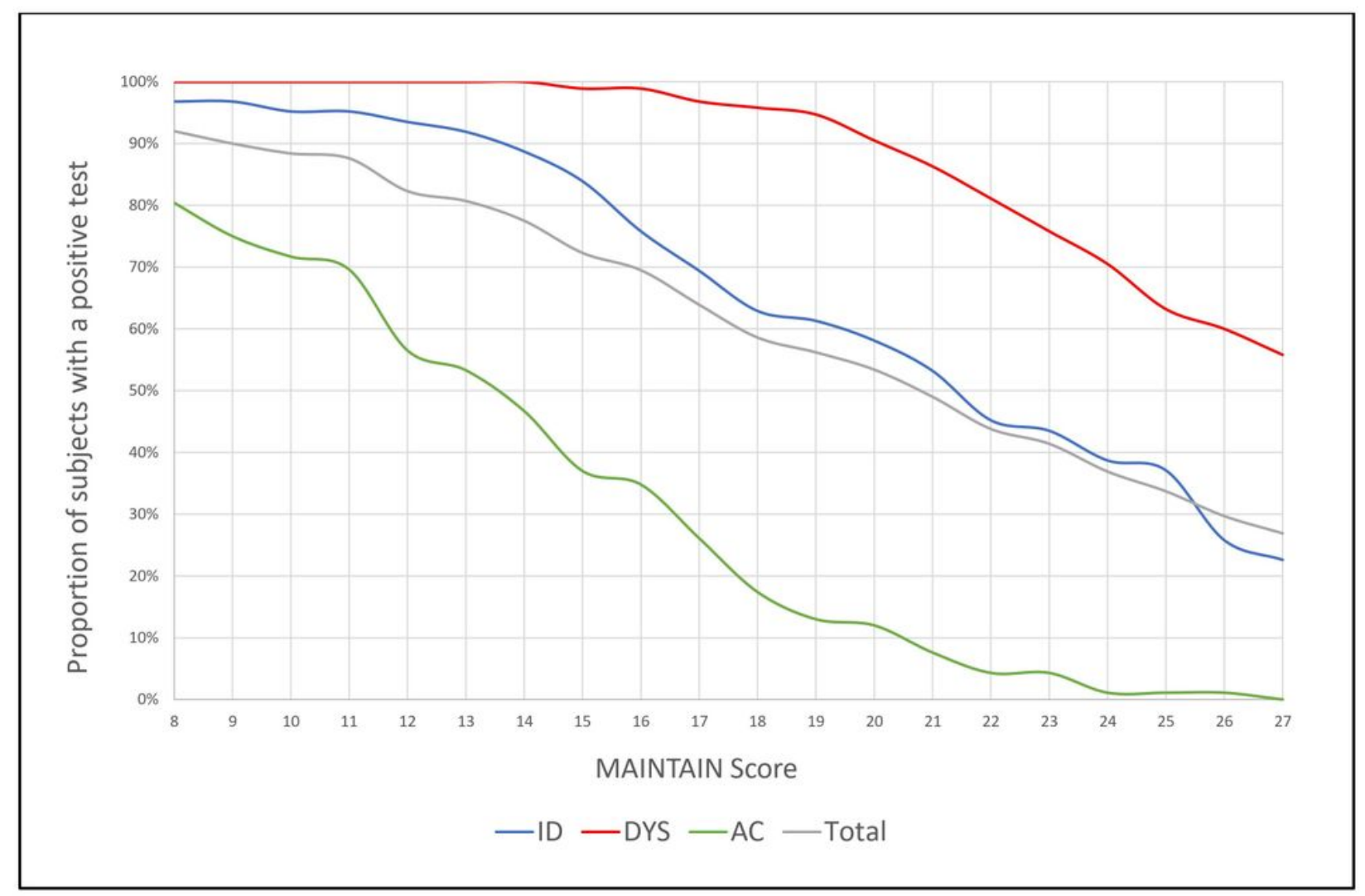

Figure 1

Proportion of individuals classified with the original MPI-S instrument with a positive test per level of the MAINTAIN instrument (MAINTAIN Score).

\section{Supplementary Files}

This is a list of supplementary files associated with this preprint. Click to download.

- Additionalfile1.pdf 